\title{
ANALISIS MICROBIOLOGICO EN YOGURT CON PROBIOTICOS
}

\author{
(Microbiological analysis in yogourt with probiotics)
}

\author{
Vallejo, F. \& Toro, M.A. \\ Universidad de Valparaíso, Escuela de Medicina, \\ Cátedra de Micología. Casilla 92 V, Valparaíso, Chile.
}

Palabras clave:Probioticos, yogurt, microbiología, bacterias lácticas

Key words: Probiotics, yogurt, microbiology, lactic acid bacteria

\section{RESUMEN}

Se estudió la calidad microbiológica de 11 muestras de un producto lácteo comercial (yogurt con probióticos), desde supermercados y otros locales afines. Se investigó la presencia de Salmonella spp., Escherichia coli y hongos levaduriformes y filamentosos, mediante la metodología estandarizada recomendada en la literatura y servicios de salud pública. El recuento de lactobacilos presentes se efectó mediante técnicas de dilución en placa con agar MRS de doble capa y la identificación de lactobacilos presente en el producto aplicando el API-50CHL.

Los resultados permitieron establecer que el producto se encuentra libre de contaminación de Salmonella spp., E.coli y hongos, sin embargo, el número de Lactobacillus casei var. rhamnosus viables, fue menor $\left(10^{5}\right)$ que el indicado en la literatura para obtener efectos benéficos en la salud.

\section{INTRODUCCION}

Los beneficios de la correcta alimentación van más allá del adecuado aporte energético y la prevención de enfermedades carenciales. Cada día se insiste más en la importancia del adecuado equilibrio en el aporte de calorías provenientes de las proteínas, grasas e hidratos de carbono y de oligoelementos, como una manera de favorecer el crecimiento óptimo y para prevenir enfermedades crónicas tales como el cáncer, trastornos cardiovasculares y otras que se asocian al aumento de las expectativas de vida de la población (1).

Un elemento que ha ido adquiriendo creciente importancia en el contexto de la alimentación infantil es el uso de los prebióticos y los probióticos $(2,12,13,14,15,24,25)$
ABSTRACT

The microbiological quality of 11 samples of a commercial lactic product (yogurt with probiotics)sold in supermarkets and other related shops was studied. The presence of Salmonella spp., Escherichia coli as well as yeast-like and filamentous fungi was examined by means of the standarized methodology suggested in the literature and in the public health services. The counting of prevailing lactobacilli was carried out with the double-layer MRS agar plate dilution technique while the identification of the former was developed by the API-50CHL method.

Results revealed that the product was not contaminated with Salmonella spp.,E.coli or fungi, yet the number of viable Lactobacillus casei var. rhamnosus was lower $\left(10^{5}\right)$ than the one designed in the literature in order to get good effects on the health.

a los que cada día se le asignan más propiedades benéficas, especialmente en la capacidad de disminuir la colonización intestinal por bacterias potencialmente patógenas y por este mecanismo, disminuir las infecciones del tracto gastrointestinal.

Los alimentos con probióticos contienen bacterias vivas que deben mantenerse estables y viables durante el almacenamiento del producto y en número suficientemente elevado que permita sobrevivir a las barreras defensivas naturales y al ecosistema del hospedador y que al ser ingeridas muestren efectos beneficiosos tanto en la prevención como en el tratamiento de desordenes gastrointestinales, tales como: la diarrea infecciosa, diarrea por tratamiento antibiótico, diarrea del viajero entre otras $(3,9,16,17,20,21,22,24,26)$. Los probióticos más co- 
múnmente utilizados hasta ahora, corresponden a bacterias del ácido láctico, de las cuales se seleccionó especialmente el Lactobacillus GG (= L.casei var. rhamnosus) por su resistencia a la acidez gástrica y a las sales biliares y por su habilidad para colonizar el intestino humano (14). Otras bacterias utilizadas son Bifidobacterium, Entero-coccus faecium (18) y levaduras tales como Saccha-romyces boulardii $(2,3,15,23)$.

Los prebióticos son componentes dietéticos tales como carbohidratos específicos que pueden ser utilizados selectivamente como nutrientes por las especies probióticas y con ello aumentar su número, previniendo de esta manera la colonización del intestino por probables patógenos $(24,26)$.

En nuestro país el consumo de yogurt y productos lácteos ha aumentado enormemente en los últimos 12 años, destinándose en 1998 un 30 \% de la producción total de leche a la elaboración de yogurt (11), por lo tanto este tipo de alimento ha incrementado su importancia en los hábitos alimentarios de nuestra población tanto adulta como infantil. A lo anterior, se suma la amplia difusión sobre el término probiótico tanto en la población general como en el área médica $(12,13)$.

Por otro lado la ingestión de alimentos contaminados plantea tal vez, un problema sanitario generalizado en nuestros días, que constituye una causa importante de reducción de la productividad económica. Actualmente se reconoce que la contaminación de los alimentos por patógenos biológicos representa un importante problema de salud pública en el mundo entero $(6,7,8,9)$.

Por los antecedentes mencionados, se analizó la calidad microbiológica de un producto lácteo (yogurt) destinado a la población general.

\section{MATERIALES Y METODOS}

\section{Muestreo}

Se seleccionaron y procesaron 11 muestras de un yogurt con probiótico de diferentes establecimientos comerciales, cinco de estas presentaban una misma fecha de vencimiento, representando un lote de producción (en este caso y debido a que el producto no rotulaba número de lote ni fecha de elaboración, un lote de producción fue definido por la fecha de vencimiento). Con el resto de las muestras se procesaron fechas de vencimiento diferentes, de tal manera que correspondieran a lotes diferentes (5). Todas las muestras fueron procesadas en duplicado.

La recolección de muestras abarcó varios establecimientos comerciales, intentando reproducir las mismas alternativas que tienen los usuarios al momento de decidir adquirir un producto (supermercados, negocios de barrio, etc.). Antes de procesarse, las muestras se almacenaron a $5^{\circ} \mathrm{C}$ por un período no mayor de tres días.

\section{Análisis de las muestras}

Para el procesamiento de las muestras se utilizó como guía las indicaciones incluidas en el Manual de Técnicas Microbiológicas para alimentos y aguas del Instituto de Salud Pública de Chile. (10)

Debido a que el yogurt tiene un $\mathrm{pH}$ menor que 4.5 se tamponó $100 \mathrm{ml}$ del producto con $100 \mathrm{ml}$ de buffer fosfato a pH 7. De esta solución se extrajeron $20 \mathrm{ml}$ que se mezclaron con $80 \mathrm{ml}$ de agua peptonada al $0.1 \%$ para tener una solución de $10^{-1}$ y de ésta última se extrajeron $10 \mathrm{ml}$ para ser mezclada con $90 \mathrm{ml}$. de agua peptonada al $0.1 \%$ para la dilución $10^{-2}$ (para la búsqueda de hongos y las bacterias Escherichia coli y Salmonella spp.). Para la búsqueda y registro de Lactobacillus, se continuaron realizando diluciones hasta llegar a $10^{-10}$.

Para la detección de E.coli se sembró $1 \mathrm{ml}$ de las diluciones $10^{-1}$ a $10^{-5}$ en $15 \mathrm{ml}$. de agar Bilis Rojo Verde Brillante, con técnica de doble capa incubando a $35^{\circ} \mathrm{C}$ por 48 horas.

Para la detección de Salmonella spp. se mezclaron $25 \mathrm{ml}$. del producto sin diluir con $225 \mathrm{ml}$. de agua peptonada incubando a $35^{\circ} \mathrm{C}$ por 18 a 24 horas, transfiriendo luego $0.1 \mathrm{ml}$. del homogeneizado a $10 \mathrm{ml}$. de caldo Rappaport Vassiliades, incubando a $43^{\circ} \mathrm{C}$ por 24 horas y finalmente se sembró por aislamiento en agar $\mathrm{SS}$ a $35^{\circ} \mathrm{C}$ por 48 horas. Se observó la aparición de cualquier colonia sospechosa y se prepararon pruebas bioquímicas para su confirmación.

Para la detección de hongos levaduriformes y filamentosos se sembró $1 \mathrm{ml}$ de las diluciones $10^{-1}$ a $10^{-}$ 5 en agar Glucosa Sabouraud Acidificado y Agar Malta incubando a $27^{\circ} \mathrm{C}$ por 7 días.

Para la detección de Lactobacillus casei var. rhamnosus se sembró $1 \mathrm{ml}$. de las diluciones $10^{-1}$ hasta $10^{-10}$ en agar MRS con técnica de doble capa, incubando a $37^{\circ} \mathrm{C}$ por 48 horas, procediendo al recuento de colonias de acuerdo al Manual del ISP. Para el aislamiento de las colonias se sembró en caldo MRS con campana incubando por 24 a 48 horas, inoculando luego en placas con agar MRS sembradas en superficie y cultivadas en anaerobiosis por 48 horas.

Se seleccionaron las colonias bajo el siguiente criterio: A los bacilos gram positivos y catalasa negativa se les aplicó el sistema API 50 CHL, de acuerdo a las instrucciones del fabricante (BIO-MERIEUX) incubando a $37^{\circ} \mathrm{C}$ por 48 horas y realizando lectura de las celdas a las $12,24 \mathrm{y}$ 48 horas.

\section{RESULTADOS}

La calidad microbiológica de los productos analizados fue definida para el presente trabajo de acuerdo al 
Reglamento Sanitario de los Alimentos vigente desde el año 1997 el que en su artículo $\mathrm{N}^{\mathrm{0}} 101$ determina lo que se considera contaminación (5).

Los $\mathrm{pH}$ de todos los lotes analizados presentaron un rango que fluctuó entre 3.61 a 3.81. No hubo en ningún lote procesado desarrollo de Escherichia coli ni Salmonella sp. a las 48 horas de cultivadas, ni tampoco hubo desarrollo de hongos levaduriformes o filamentosos a los siete días de cultivo en ninguna de las placas sembradas.

En todas las siembras realizadas de las diversas muestras, los recuentos de UFC/ml adecuados para la lectura de colonias bacterianas correspondieron a las diluciones $10^{-4}$ y la $10^{-5}$, disminuyendo el número de colonias en las diluciones mayores, siendo nulo en las diluciones superiores a $10^{-7}$ (Cuadro 1 ). Se destaca que la muestra 1 representa el promedio de 5 muestras por lote, las restantes, el promedio de dos muestras.

El sistema de identificación bacteriana (API 50-CHL) nos permitió identificar con certeza que el probiotico utilizado corresponde al Lactobacillus casei var. rhamnosus (ATCC 53103).

\section{DISCUSION}

El papel que la microbiota intestinal juega en la metabolización de los alimentos es conocido hace mucho tiempo. La incorporación de los antibióticos al arsenal médico ha venido a reforzar la importancia de los organismos comensales y es ampliamente conocida la diarrea por tratamiento antibiótico.

El rol inmunomodulador de la microbiota es un concepto relativamente nuevo, que se ha venido estudiando en las últimas dos décadas(29). El desarrollo de nuevos métodos de investigación (citometría de flujo y uso de anticuerpos monoclonales entre otros), ha servido para investigar la asociación entre los alimentos y algunas enfermedades. Un desbalance en el ecosistema intestinal ha sido descrito en numerosas enfermedades entre las que se

\section{Cuadro No 1.- Recuento de Lactobacillus casei var. rhamnosus según muestra.}

\begin{tabular}{|c|c|c|}
\hline $\begin{array}{c}\mathbf{N}^{\circ} \\
\text { muestras }\end{array}$ & Dilución & $\begin{array}{c}\text { Recuento } \\
\text { (ufc/ml.) }\end{array}$ \\
\hline 1 & $10-5$ & 32 \\
\hline 2 & $10-4$ & 27 \\
\hline 3 & $10-4$ & 35 \\
\hline 4 & $10 . .-4$ & 247 \\
\hline 5 & $10 .-4$ & 56 \\
\hline
\end{tabular}

decriben: la enfermedad inflamatoria intestinal, artritis reumatoidea, diarrea por rotavirus y enfermedades atópicas (31). En la actualidad existe evidencia que asocia alteraciones de estos organismos con el desarrollo de atopia. Niños con enfermedad atopica clinicamente manifestada a los dos años de edad presentan en los cultivos fecales una menor colonización por Lactobacillus y mayor cantidad de coliformes y Staphylococcus aureus en comparacion con niños sin enfermedad atópica (35).

La introducción de bacterias ácido lácticas a la industria alimentaria marcó un paso hacia el desarrollo de nuevas modalidades terapéuticas relacionadas con las modificaciones alimentarias. Se acepta que los alimentos tienen un rol que va mas alla del placer gastronómico y del aporte de energía y de nutrientes al organismo. Actualmente se habla de "alimentos funcionales”, refiriéndose a aquellos que son utilizados para mejorar ciertas funciones fisiológicas de manera de prevenir o mejorar la calidad de vida(30). Entre estos se incluyen los probióticos y prebióticos.

El uso de probióticos, principalmente de Lactobacillus y Bifidobacterium, ha sido estudiado en ensayos clínicos, siendo las principales enfermedades tratadas la diarrea aguda infantil, la diarrea asociada a antibióticos y la diarrea del viajero(1,2,16,17,18, 20,22,31,32). Otros reportes muestran que sujetos con dietas ricas en probióticos presentan un aumento de la respuesta inmune innata (29), aumento en la expresión de receptores del complemento y de moléculas de adhesión en neutrofilos y monocitos (33) y un incremento de interleukina 10 en los linfocitos de la circulación periférica (34). Lo anterior llevaría al desarrollo de una respuesta inmune que evitaría eventos inflamatorios alérgicos, probablemente mediada por la estimulación de linfocitos T tipo 3 (TH3), importantes en la secreción de citoquinas antinflamatorias.

Existen diversas formas de administración de probióticos ensayadas hasta ahora $(14,19.22)$. En nuestro país una industria productora de leches prefirió su inclusión en leches fermentadas, siendo la primera bacteria escogida Lactobacillus casei var. rhamnosus (conocido como Lactobacillus GG [ATCC 53103]) y al momento de desarrollar el presente trabajo otra industria de alimentos estaba introduciendo un producto conteniendo Lactobacillus johnsonii (anteriormente conocido como Lactobacillus acidophilus).

Los análisis efectuados en este estudio nos permitieron asegurar en el yogurt analizado la ausencia de contaminación con bacterias potencialmente patógenas así como la ausencia de desarrollo de hongos, algunos capaces de producir micotoxinas. Es importante destacar que el $\mathrm{pH}$ al que se expende el producto es un factor inhibitorio para el crecimiento de bacterias no ácido lácticas $(4,12)$. 
Las propiedades terapéuticas de los probióticos dependen de la capacidad de las bacterias para sobrevivir a la acidez gástrica y a la acción de las enzimas y de las sales biliares. El segundo elemento que debe considerarse es la capacidad de estas bacterias para colonizar el intestino humano en forma permanente. Las cepas ensayadas clínicamente hasta ahora han demostrado ser capaces de resistir las barreras naturales del sistema digestivo pudiendo ser recogidas en las deposiciones de voluntarios sanos, pero no hubo una colonización permanente $(32,36)$. Esto último destaca la importancia de que un alimento con probióticos contenga un número de bacterias lo suficientemente elevado al momento de ser ingerido. $(28,33)$. Se ha establecido que el número "mínimo terapéutico" de bacterias viables deseable es de $10^{7} \mathrm{UFC} / \mathrm{ml}$ de producto final, pero para que se desarrolle un efecto terapéutico evidenciable en el tiempo el alimento debe ser consumido diariamente y debe contener una cantidad de bacterias vivas equivalentes a $10^{8} \mathrm{UFC} / \mathrm{ml}(12,15,19,32)$. En la revisión de la literatura no hay ensayos clínicos que muestren beneficios con concentraciones menores a las indicadas.

Los cultivos de Lactobacillus GG permitieron identificar envases con recuentos entre las diluciones $10^{-4}$ a $10^{-5}$ tal como lo muestra el Cuadro $\mathrm{N}^{\circ} 1$, en tanto el desarrollo de Lactobacillus en las diluciones $10^{-6}$ o superiores fue mínimo o nulo. La rotulación del envase indica que "el número de bacterias vivas al momento del envasado es de $10^{9}$ ". Lo aconsejable era efectuar los análisis lo mas próximo a esta fecha, pero debido a que sólo se rotula la fecha de vencimiento esto último no fue posible evaluarlo.

Nighswogder (1999), analizó muestras de Lactobacillus $G G$ en mantequilla y yogurt, encontrando que existía una pérdida de la viabilidad durante el período de almacenamiento y que en algunas muestras esta dismi-

boulardii. Pediatr Res. ;20: 192-296

4.- Kilic, A. \& Pavlona, S. (1996). Analysis of Lactobacillus, Phage and bacteriocins in American diary products and Characterization of a Phage Isolated from yogurt App and Enviromental Microb.; 62: 2111-2116

5.-Nuevo Reglamento Sanitario de los Alimentos. (1982). Editorial Cumbres, Santiago

6.- Hannesey, T.; Hedberg, C.; Slutker, L. et al. (1996) A national outbreak of Salmonella enteritidis infections from ice cream. N Engl J Med.; 334: 1281-1286

7.- Desenclos, J-C.; Bouvert, P.; Benz-Lemoine, E. et al.. (1996). A case, finding and epidemiological study. BMJ. ; 312: 9194 nución era realmente significativa (27). Es posible que los cultivos desarrollen ácidos orgánicos u otros compuestos inhibitorios en las leches fermentadas que pudieran influir en la sobrevida de algunos probióticos (28). El análisis en detalle de nuestros resultados nos permitió apreciar que mientras más cerca es la fecha de vencimiento del productos, es decir mas lejano a la fecha de elaboración, más bajo es el recuento obtenido (dato no mostrado).

En resumen, podemos concluir que los productos analizados se encuentran libres de contaminación por Salmonella sp., Escherichia coli y hongos levaduriformes y filamentosos. Sin embargo, podemos señalar que el número de Lactobacillus GG viables al momento del análisis no correspondería a lo descrito en la literatura como mínimo necesario para obtener efectos beneficiosos para la salud. Son necesarios más estudios para analizar el impacto del período de almacenamiento en la sobrevida de este producto.

\section{Agradecimientos}

Se agradece los comentarios y la valiosa colaboración del Dr. Miguel Zazopulos de la Universidad Técnica Federico Santa María, Sede José Miguel Carrera.

\section{REFERENCIAS}

1.- Scheppach, W.; Luehrs, H. \& Menzel, T. (2001). Beneficial health effect of low-digestible carbohydrate consumption British Journal of Nutrition; 85: suppl 1: 23S-30S

2.- Chapoy, P.(1985). Traitement des diarrhées aiguës infantiles: essai contrôlé de Saccharomyces boulardii Annales de Pediatrie; 32: $561-563$

3.- Buts, J. \& Bernasconi, P. (1986).Response of human and rat small intestinal mucosa to oral administration of Saccharomyces

8.- Rampling, A. (1996). Raw milk cheese and Salmonella BMJ.; 312: $67-68$

9.- Outbreak of Eschirichia coli O157 infection associated with pasteurized milk supply. (Letter) Lancet. 1994; 344: 1015

10.- Ministerio de Salud, Instituto de Salud Pública de Chile. (1998). Manual de técnicas microbiológicas para alimentos y aguas. Primera Edición.

11.- Ministerio de Agricultura; Oficina de Estudios y Políticas Agrarias. Abril de 1999Boletín de la leche..

12.- Lee, Y. \&. Salminen, S. (1995). The coming of age of probiotics Trends in Food Science \& Tecnology. Vol 6 july

13.- Levy, J. (1998). Inmunonutrition: The pediatric experience Nutrition 14: 641-647 
14.- Guerin-Danan, C.; Chabanet, C.; Pedone, C. et al. (1998). Milk fermented with yogurt cultures and Lactobacillus casei compared with yogurt and gelled milk: influence on intdestinal microflora in healthy infants. Am. J. Clin.Nutr. 67: 111-117

15.- Berg, R.; Bernasconi, P. \& Fowler, D. (1993). Inhibition of Candida albicans translocation from the gastrointestinal tract of mice by oral administration of Saccharomyces boulardii. J. Infect. Dis. 168: $1314-1318$

16.- Katerlaris, P.; Salam, I. \& Farthing, M. (1995). Lactobacilli to prevent traveler's diarrhoea? Engl. J. Med. 16: 1360-1361

17.- Oksanen, P.; Salminen, S. \& Saxelin, M. (1990). Prevention of traveler's diarrhoea by Lactobacillus GG Ann. Med. 22: $53-56$

18.- Saavedra, J.; Bauman, N.; Oung, I. (1994). Feeding of Bifidobacterium bifidum and Streptococcus thermophilus to infant in hospital for prevention of diarrhoea and shedding of rotavirus. Lancet 344: 1046-1049

19.- Djouzy, Z.; Andrieux, C. \& Degivry, M. (1997). The association of Yogurt starters with Lactobacillus casei DN 114.001 in fermented milk alters the composition and metabolism of intestinal microflora in germ-free rats and in human flora-associated rats. J. Nutr. 127: 2260-2266

20.- Sepp, E.; Tamm, E.; Torm, S. et al. (1995). Impact of Lactobacillus probiotics on the faecal microflora in children with shigellosis. Microecology and Therapy 23:74-80

21.- Majamaa, H.; Isolauri, E. \& Saxelin, M. (1995).Lactic acid bacteria in the treatment of acute rotavirus Gastroenteritis. J. Ped .Gastroent. and Nutr. 20: 333-338

22.- Biller, J.; Katz, A.; Flores, A. et al. (1995).Treatment of recurrent Clostridium difficile colitis with Lactobacillus GG. J. Ped. Gastroent. and Nutr. 21: 224-226

23.- Höchter, H.; Chase, D. \& Hagenhoff G. (1990). Saccharomyces boulardii in acute adult diarrhoea. Münch. Med. Wschr. 132: 188-192

24.- Gibson, G. \& Fuller, R. (2000). Aspect of in vitro and in vivo research approaches directed toward identifying Probiotics and Prebiotics for human use. J .Nutr. 130: 391S-395S
25.- Heller, J. (2001). Probiotic bacteria in fermented foods: product characteristics and starter organisms . Am. J. Clin. Nutr. 73: 374S3795

26.- Schrezenmeir, J. \& de Vrese, M.(2001). Probiotics, Prebiotics and synbiotics - approaching a definition . Am. J. Clin. Nutr. 73: 361S-364S

27.- Nighswonger, B.; Brashears, M. \& Guilliland, S. (1996). Viability of Lactobacillus acidophilus and Lactobacillus casei in fermentede milk products during refrigerated storage. J. Diary Sci. $79: 212-219$

28.- Tuomola, E.; Critteden, R.; Playne, M.; Isolauri, E.;Salminen, S. (2001). Quality assurance criteria for probiotic bacteria. Am. J. Clin. Nutr. 73:393S-398S

29.-Matsuzaki, T. \& Chin, J. (2000). Modulating immune responses with probiotic bacteria. Immunology and cell biology 78:67-73

30.-Lopez-Varela, S.; Gonzalez-Gross, M. \& Marcos, A. (2002). Functional food and the immune system: a review. European Journal of Clin. Nutrition 56 Suppl 3: 529-533

31.- Rautava, S. \& Isolauri, E. (2002). The development of gut immune responses and gut microbiota: effects of probiotics in prevention and treatment of allergic disease. Curr. Issues Intest. Microbiol. 3:15-22

32. Gotteland, M. (1999). Probioticos: microorganismos de la dieta al servicio de la Salud . Rev. Chil. Nutr. 26:20-33

33.-Pelto, L.; Isolauri, E.; Lilius, E.; Nuutila, J.; Salminen, S. (1998). Probiotic bacteria down regulate the milk-induced inflamatory response in milk-hypersensitive subjects but have an immunostimulatory effect in helathy subject . Clin. Exp. Allergy 28:1474-1479

34.- Pessi, T.; Sutas, Y.; Hurme, M.; Isolauri, E. (2002) Interleukin-10 generation in atopic children following oral Lactobacillus rhamnosus GG. ). Clin. Exp. Allergy 10: 1804-1808

35.- Bjorksten, B.; Naaber, P.; Sepp, E.; Mikelsar, M. (1999) The intestinal Microflora in allergic Estonian and Swedish 2-yearsold children. Clin. Exp. Allergy 29: 342-346

36.-Bouhnic ,Y.; Pochart, P.; Marteau, P.; Arlet, G.; Goderel, Y.; Rambaud, J.C. (1992). Fecal recovery in humans of viable Bifidobacterium sp. ingested in fermented milk. Gastroenterology. 102: $875-878$ 